\title{
Research on the Impediment to Vibration Wave Propagation of Pipe from a vibration isolation mass
}

\author{
Gong Yufeng ${ }^{1, *}$, Peng Weicai ${ }^{1}$, Zhang Junjie $^{1}$ and Liu Zhizhong ${ }^{1}$ \\ ${ }^{1}$ National Key Laboratory on Ship Vibration \& Noise, China Ship Development and Design Center, Wuhan 430064, China
}

\begin{abstract}
In order to control the pipe vibration, the performance of flexible connection with vibration isolation mass in impeding vibration wave propagation is studied. Based on the principle of impedance mismatching and numerical analysis method, the influences of vibration isolation mass and rotational inertia on the vibration wave propagation in pipe were discussed. The results show that the isolation mass is good at reducing the vibration of wave transmission in intermediate and high frequency domain. Meanwhile, The larger rotational inertia of the isolation mass, the better the damping effect. A useful reference was provided for the application of flexible connection to the vibration isolation and noise reduction of ship pipe.
\end{abstract}

\section{Introduction}

With the advancement of science and technology, the number of equipments installed in ships and submarines are increasing. The vibration propagated through the piping system has a significant effect on the acoustic stealth performance of the ship and submarine. In order to control the pipe vibration, researchers proposed various vibration reduction method of pipe. Vibration isolation mass is one of the main vibration reduction method, it has been widely applied in the ship and submarine. Research on the characteristics of pipe vibration impeded by vibration isolation mass can help the engineer to design the vibration isolation mass of pipe, reduce the structural vibration propagated through the piping system, and improve the acoustic stealth performance of the vessel.

Vibration isolation mass mainly causes the impedance mismatch by leading to the discontinuity of the mass or rigidity of the steady structure, thereby reflecting the incident wave effectively. Researchers conducted a large number of theoretical, numerical, and experimental studies on the mechanism and characteristic of vibration isolation mass [1-3]. Cremer et al [4] briefly discussed the vibration isolation mass, and proposed that the vibration isolation mass impedes the vibration wave propagation by changing the local rigidity of the steady structure. However, the influence of its geometry parameters on the vibration wave propagation was not specially studied. Yao et al [5] discussed the research progress on vibration isolation mass, as well as the characteristic of vibration propagated in the discontinuous structure. Liu et al [6] studied the impediment to structure-borne sound transmission from rigid vibration isolation mass when the infinite plate is motivated by concentrated force, and the feasibility of simplified vibration isolation coefficient formula was testified by theoretical analysis, of which the availability was demonstrated by calculation model and experimental study. Ling and $\mathrm{Wu}$ [7] has studied the impediment to structure-borne sound transmission from vibration isolation mass by finite element method. Their research results show that the multi-stage vibration isolation mass has a good impediment to structure-borne sound transmission.

The above research indicates that the vibration isolation mass could impede the vibration wave propagation in the plate effectively. For the application of vibration isolation mass in the pipe, Yang et al [8] studied the characteristic of vibration propagated in fluid-filled pipe and the vibration reduction effect of vibration isolation mass on the fluid-filled pipe by the experimental method. Meanwhile, $\mathrm{He}$ and $\mathrm{He}$ [9] studied the characteristic of vibration wave propagated in infinite pipe by the theoretical method. Their research result indicates that the transmission loss in the pipe structure is mainly related to the mass and rotational inertia of vibration isolation mass.

Based on the principle of impedance mismatching and numerical analysis method, the influences of vibration isolation mass and rotational inertia on the vibration wave propagation in pipe were discussed in this paper. Some useful reference were provided for the engineering to design the pipe system of ship and submarine.

\section{Theoretical basis of pipeline vibration isolation mass}

\footnotetext{
* Corresponding author: Gong_yu_feng@163.com
} 
Current research results $[8,9]$ show that there are many forms of vibration wave in the pipe, such as longitudinal wave, transverse wave and bending wave. due to the bending stiffness of structure is usually smaller than the longitudinal and transverse stiffness, the structure is prone to bending deformation. Under the same external force, the bending deformation of structure is larger. thus, the vibration of the pipe is mainly caused by the bending waves propagating in the pipe. Based on the existing theories, the effect of vibration isolation mass on the propagation characteristics of bending waves in the pipe is discussed.

Assume that there is a vibration isolation mass in an infinite pipe, as shown in Fig. 1. Bending ware propagate in the pipe form left to right. The mass of the vibration isolation mass is $m$, the rotational inertia of the vibration isolation mass is $J$.

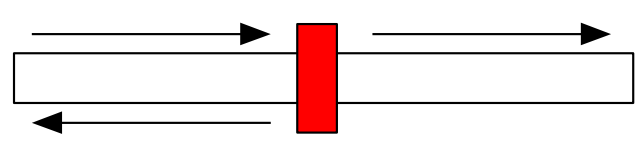

Fig. 1. Schematic of a vibration isolation mass in an infinite pipe

According to the equation of Euler-Bernoulli, the equation of bending wave could be expressed by:

$$
\frac{E I}{\rho S} \cdot \frac{\partial^{4} w}{\partial X^{4}}=-\frac{\partial^{2} w}{\partial t^{2}}
$$

where $w$ is the bending deformation of pipe, $E$ is the elastic modules of pipe, $I$ is the moment of inertia of pipe, $\rho$ is the density of pipe, $S$ is the cross-section area of pipe, $t$ is time.

The solution of eq.(1) can be expressed by eq.(2)

$$
w=A e^{k x} e^{j \omega t}+B e^{-k x} e^{j \omega t}+C e^{j k x} e^{j \omega t}+D e^{-j k x} e^{j \omega t}
$$

where $A, B, C, D$ is the complex constant, $\omega$ is the angular frequency, $k$ is the wave number.

the following expression could be obtained by satisfying the condition of continuous displacement and continuous displacement gradient, Newton's second law and moment balance.

$$
\begin{gathered}
\left.w_{L}\right|_{X=0}=\left.w_{R}\right|_{X=0} \\
\left.\frac{\partial w_{L}}{\partial X}\right|_{X=0}=\left.\frac{\partial w_{R}}{\partial X}\right|_{X=0} \\
\left.f_{L}\right|_{X=0}-\left.f_{R}\right|_{X=0}=\left.m\left(\frac{\partial^{2} w_{R}}{\partial t^{2}}\right)\right|_{X=0} \\
\left.M_{L}\right|_{X=0}-\left.M_{R}\right|_{X=0}=\left.J\left(\frac{\partial^{3} w_{R}}{\partial x \partial t^{2}}\right)\right|_{X=0}
\end{gathered}
$$

The location of vibration isolation mass is $X=0 . w_{\mathrm{L}}$ and $w_{\mathrm{R}}$ is the bending deformation on left and right side of vibration isolation mass. $f_{\mathrm{L}}$ and $f_{\mathrm{R}}$ is the shear force on left and right side of vibration isolation mass. $M_{\mathrm{L}}$ and $\mathrm{M}_{\mathrm{R}}$ is the bending moment on left and right side of vibration isolation mass.

Shear force and bending moments on both sides of vibration isolation mass can be expressed by:

$$
\begin{gathered}
f=E I \frac{\partial^{3} w}{\partial X^{3}} \\
M=-E I \frac{\partial^{2} w}{\partial X^{2}}
\end{gathered}
$$

Substituting the above formula into Eq.(3) to Eq.(6), then, the transmission loss in the pipe could be obtained by combining with Eq.(2).

$$
\begin{gathered}
T L=10 \lg \left(\frac{\left(4+\alpha-\alpha_{J}-\frac{1}{2} \alpha \alpha_{J}\right)^{2}+\left(\alpha+\alpha_{J}\right)^{2}}{\left(4+\alpha-\alpha_{J}\right)^{2}}\right) \\
\alpha=\frac{m \omega^{2}}{E I k^{3}}=\frac{m}{\rho \cdot S} \cdot \frac{2 \pi}{\lambda} \\
\alpha_{J}=\frac{J \omega^{2}}{E I k}=\frac{J}{\rho \cdot S} \cdot k^{3}
\end{gathered}
$$

It could be known from Eq.(9) that the transmission loss of bending wave in the pipe is mainly related to the mass and rotational inertia of vibration isolation mass. however, it should be noted that the main parameter for impeding the vibration wave is the ratio of the mass of vibration isolation mass to the mass of pipe, rather than the absolute mass of the vibration isolation mass.

\section{Numerical analysis}

\subsection{Numerical validation}

Before the numerical analysis of pipeline vibration isolation mass, the numerical method which to be adopted is verified by the experimental results presented by Wang et al [10].The experimental model of pipeline vibration isolation mass presented by Wang et al [10] is shown in Fig 2(a). The outer radius of steel circular hollow pipe is $24 \mathrm{~mm}$. The inner radius of steel circular hollow pipe is $21 \mathrm{~mm}$. The thickness of pipe is $3 \mathrm{~mm}$. Pipe is fixed by pipe clamps. The vibration isolation mass is simulated by a metal flange in the experiment. The outer radius of flange is $33.5 \mathrm{~mm}$. The inner radius of flange is $24 \mathrm{~mm}$. The thickness of flange is 11.3 $\mathrm{mm}$. The hammer is struck on the right side of the pipe to generate vibration. Then the vibration wave propagates along the pipe to the left side. Due to the influence of vibration isolation mass, the vibration wave will have a transmission loss in the left side of pipe. The point 1 is the reference point, and the vibration level drop is determined by the difference of acceleration level between point 1 and point 2 .

In order to validate the numerical method adopted in this paper, the numerical simulation of the experiment has been conducted by the general commercial finite element software MSC.Patran, as shown in Fig 2 (b).

The comparison of vibration level drop between point 1 and point 2 obtained by numerical method and experiment measurement is shown in Fig 3. It could be 
observed that the result obtained by numerical method is agree well with the result obtained by experiment measurement. The adopted numerical method could reasonable simulation the vibration wave propagating in the pipeline.

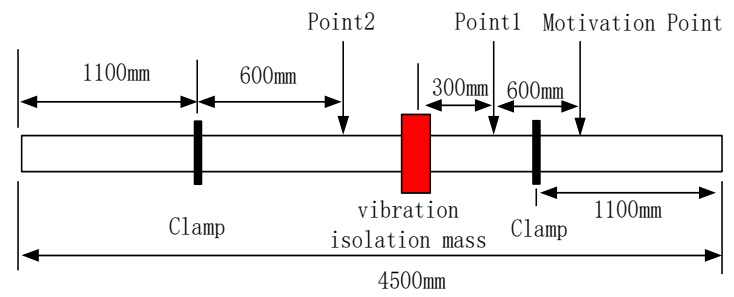

(a) Experimental model

(b) Numerical Model

Fig. 2. Schematic of experimental model and numerical model.

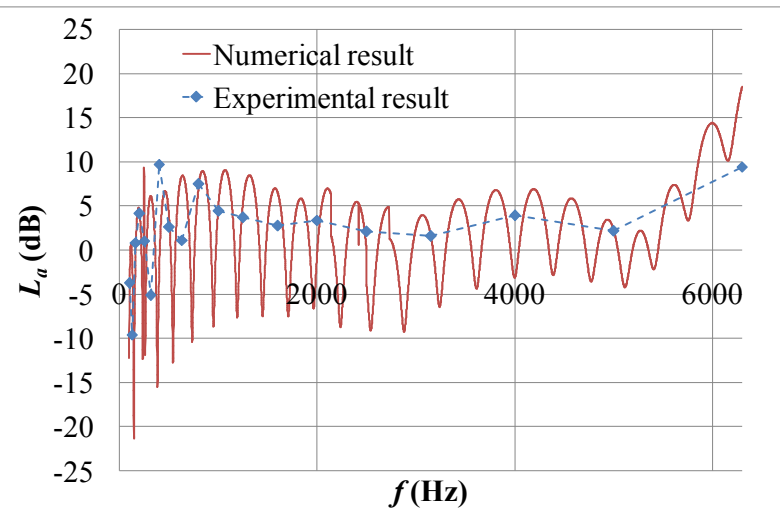

Fig. 3. Comparison of level difference between experimental measurement and numerical calculation (the blue marker is the experimental data, the blue dash line is the trend line).

\subsection{Numerical model}

Based on the commercial finite element software MSC.Patran, the model of vibration isolation mass and pipe is established. The influence of vibration isolation mass on the vibration attenuation of pipe have been studied by the finite element method. The schematic of vibration isolation mass and pipe is shown in Fig.4. Equipment is simulated by point mass. Pipe is simulated by beam element. Flexible connection and clamp are simulated by linear spring element.

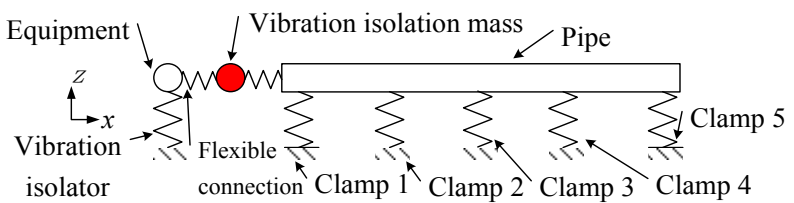

Fig. 4. Schematic of calculation model.
The density of pipe is $\rho=7.8 \times 10^{3} \mathrm{~kg} / \mathrm{m}^{3}$, and the elastic modules and Poisson ratio is $E=2.1 \times 10^{11} \mathrm{~Pa}$ and $\nu=0.3$. The axial and radial dynamic stiffness of flexible connection is $k_{\mathrm{a}}=3.7 \times 10^{4} \mathrm{~N} / \mathrm{m}$ and $k_{\mathrm{r}}=2.5 \times 10^{4} \mathrm{~N} / \mathrm{m}$, and the torsional dynamic stiffness of flexible connection is $k_{\mathrm{t}}=3.7 \times 10^{4} \mathrm{~N} / \mathrm{m}$. The mass of equipment is $m_{\mathrm{e}}=1000 \mathrm{~kg}$. The dynamic stiffness of vibration isolator $\mathrm{s}$ which are arranged under the equipment in all direction are $k=3.954 \times 10^{5} \mathrm{~N} / \mathrm{m}$. The length of pipe is $L=5 \mathrm{~m}$, and 5 clamp are set at equal intervals on the pipe. The exciting force in $x$ direction and $z$ direction, $F_{x}=F_{z}=100 \mathrm{~N}$, is applied to the point mass which simulates the equipment. The bottom of the clamp is considered to be rigid constraint in this study.

In order to compare the influence of parameters such as the mass and rotational inertia of vibration isolation mass, the dynamic stiffness of clamp, 7 examples are set up in this study. The dynamic stiffness value of clamp 1 is changed in different example. however, the dynamic stiffness value of clamp 2 to clamp 5 is remain unchanged. The specific parameters are shown in Table 1. The calculation frequency range from 1 to $500 \mathrm{~Hz}$, and the calculation frequency interval is $1 \mathrm{~Hz}$. In order to compare the vibration transmission from pipe to the hull in different examples, $F^{\mathrm{i} \Sigma}$ which has been defined in Eq.(12) is used as the comparison parameters.

$$
F^{i \Sigma}=\sqrt{\sum_{j=1}^{5}\left(F^{j}\right)^{5}}
$$

where $j$ is the number of clamp.

Table 1. Parameters of numerical examples.

\begin{tabular}{|c|c|c|c|c|}
\hline \multirow[b]{2}{*}{ Id } & \multicolumn{2}{|c|}{$\begin{array}{c}\text { Vibration isolation } \\
\text { mass }\end{array}$} & \multirow{2}{*}{$\begin{array}{c}\text { Dynamic } \\
\text { stiffness of } \\
\text { clamp 1, } \\
k_{1}(\mathrm{~N} / \mathrm{m})\end{array}$} & \multirow{2}{*}{$\begin{array}{c}\text { Dynamic } \\
\text { stiffness of } \\
\text { clamp 2 5, } \\
k_{2}(\mathrm{~N} / \mathrm{m})\end{array}$} \\
\hline & $\begin{array}{l}\text { Mass, } \\
m(\mathrm{~kg})\end{array}$ & $\begin{array}{c}\text { Rotationa } \\
\text { l inertia, } \\
J\left(\mathrm{~kg} \cdot \mathrm{m}^{2}\right)\end{array}$ & & \\
\hline 1 & 30 & 0.3 & $3.0 \times 10^{4}$ & $3.0 \times 10^{4}$ \\
\hline 2 & 30 & 1.0 & $3.0 \times 10^{4}$ & $3.0 \times 10^{4}$ \\
\hline 3 & 30 & 1.6 & $3.0 \times 10^{4}$ & $3.0 \times 10^{4}$ \\
\hline 4 & 30 & 1.6 & $1.0 \times 10^{9}$ & $3.0 \times 10^{4}$ \\
\hline 5 & 15 & 1.6 & $1.0 \times 10^{9}$ & $3.0 \times 10^{4}$ \\
\hline 6 & 5 & 1.6 & $1.0 \times 10^{9}$ & $3.0 \times 10^{4}$ \\
\hline 7 & 0 & 0 & $1.0 \times 10^{9}$ & $3.0 \times 10^{4}$ \\
\hline
\end{tabular}

\subsection{Influence of the mass of vibration isolation mass and its characteristics of vibration attenuation}

In order to study the influence of vibration isolation mass on the vibration wave propagated in pipe, the examples presented in Table. 1 have been calculated by the finite element method. The comparison of the transmission force $F^{i \Sigma}$ between the example 4 and 7 is shown in Fig.5. The example 4 is the plan that installed 
the flexible connection with vibration isolation mass, and the example 7 is the plan that installed the flexible connection with not vibration isolation mass.

According to the comparison, it could be found that the transmission force $F^{i \Sigma}$ of example 4 and 7 is similar when the frequency lower than $5 \mathrm{~Hz}$. It is because that the motion of the pipe is mainly rigid body motion when the frequency is very low [3], such as lower than $5 \mathrm{~Hz}$. whether the installation of vibration isolation mass does not have a significant effect on the transmission force of clamp, $F^{\mathrm{i \Sigma}}$.

From $10 \mathrm{~Hz}$ to the cutoff frequency $500 \mathrm{~Hz}$, the transmission force $F^{\mathrm{i} \Sigma}$ of example 4 that installed the flexible connection with vibration isolation mass is noticeable difference from the transmission force $F^{\mathrm{i}}$ of example 7 which installed the flexible connection with not vibration isolation mass. The effect of vibration isolation mass on impeding the vibration of pipe is improving with the increasing of calculation frequency. This is due to the fact that the vibration isolation mass is rigid vibration reduction method, while rigid vibration reduction method could effective impeding the propagation of medium and high frequency vibration wave.

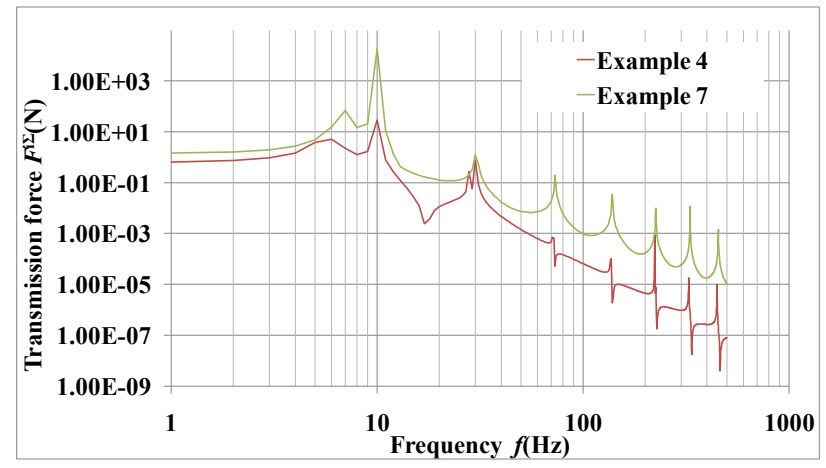

Fig. 5. Comparison of the transmission force $F^{\mathrm{i} \Sigma}$ between example 4 and 7.

In order to comparison the influence of the mass of vibration isolation mass on the vibration wave propagated in pipe, the transmission force $F^{\mathrm{i} \Sigma}$ of example 4, 5 and 6 have been shown in Fig.6. According to comparison, it is found that the transmission force $F^{\mathrm{i} \Sigma}$ of example 4,5 and 6 is similar when the frequency lower than $5 \mathrm{~Hz}$. This indicates that when the frequency is very low, such as lower than $5 \mathrm{~Hz}$, the motion of the pipe is mainly rigid body motion. The ratio betweem the mass of vibration isolation mass and the density of pipe does not have a significant effect on the transmission force of clamp, $F^{i \Sigma}$, when the motion of the pipe is mainly rigid body motion.

In addition, it could be observed that the transmission force of clamp $F^{\mathrm{i \Sigma}}$ is obvious decreasing when the mass of vibration isolation mass increased from $5 \mathrm{~kg}$ to $15 \mathrm{~kg}$. However, when the mass of vibration isolation mass increased from $15 \mathrm{~kg}$ to $30 \mathrm{~kg}$, the transmission force of clamp $F^{\mathrm{i} \Sigma}$ almost remain unchanged.

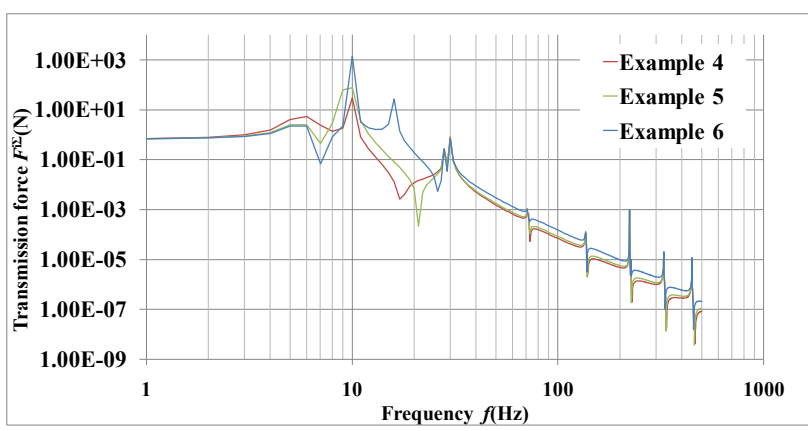

Fig. 6. Comparison of the transmission force $F^{\mathrm{i} \Sigma}$ between example 4, 5 and 6.

In order to comparison the influence of the rotational inertia of vibration isolation mass on the vibration wave propagated in pipe, the transmission force $F^{i \Sigma}$ of example 1,2 and 3 have been shown in Fig.7. According to comparison, it is found that the transmission force $F^{\mathrm{i} \Sigma}$ of example $1 \quad(I=0.3), 2(I=1.0)$ and $3(I=1.6)$ is not significant difference when the frequency lower than $5 \mathrm{~Hz}$. However, from $10 \mathrm{~Hz}$ to the cutoff frequency $500 \mathrm{~Hz}$, the transmission force $F^{\text {i }}$ of example $3(I=1.6)$ is one order of magnitude smaller than the transmission force $F^{i \Sigma}$ of example $1 \quad(I=0.3)$. It indicates that the rotational inertia of vibration isolation mass have a significant effect on impeding the propagation of medium and high frequency vibration wave.

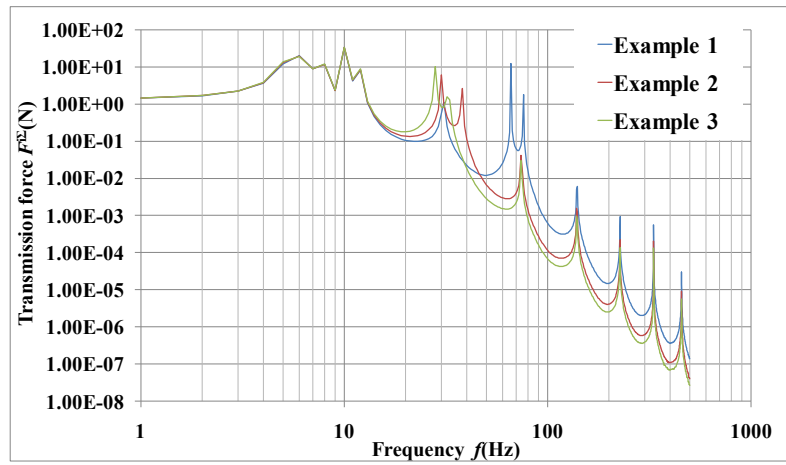

Fig. 7. Comparison of the transmission force $F^{\mathrm{i} \Sigma}$ between example 1, 2 and 3.

\subsection{Influence of the arrangement method of clamp and its characteristics of vibration attenuation}

Not only the vibration isolation mass, but also the arrangement of clamp of pipe could influence the vibration wave propagation in pipe [8]. Therefore, by changing the dynamic stiffness of the first level clamp, the influence of the arrangement method of clamp on the on the vibration wave propagated in pipe is studied. Fig. 8 shows the comparison of the transmission force $F^{\text {i }}$ between the example 3 and 4 . The first level of clamp is consider to be installed rigidly by given the dynamic stiffness $k_{1}=1.0 \times 10^{9} \mathrm{~N} / \mathrm{m}$ in example 4 . The rest level of clamp in example 4 is consider to be installed elastic by given the dynamic stiffness $k_{2}=3.0 \times 10^{4} \mathrm{~N} / \mathrm{m}$. Meanwhile, all of clamp in example 3 is consider to be installed elastic by given the dynamic stiffness $k_{1}=k_{2}=3.0 \times 10^{4} \mathrm{~N} / \mathrm{m}$. 
According to comparison the transmission force of clamp $F^{\mathrm{i} \Sigma}$, it could be found that the transmission force $F^{\mathrm{i} \Sigma}$ of example 4 is significant lower than example 3 when the frequency lower than $100 \mathrm{~Hz}$. From $100 \mathrm{~Hz}$ to the cutoff frequency $500 \mathrm{~Hz}$, the difference between the transmission force of example 3 and the transmission force of example 4 is diminishing with the increasing of frequency.

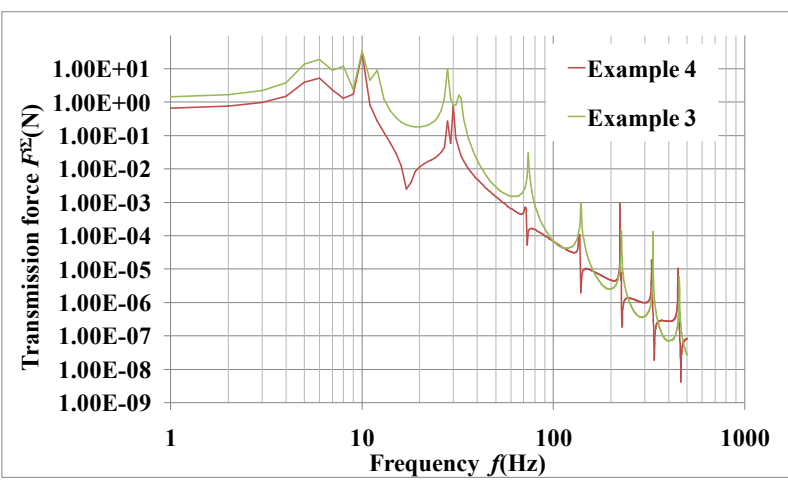

Fig. 8. Comparison of the transmission force $F^{\mathrm{i} \Sigma}$ between example 3 and 4.

Fig.9 shows the comparison of the transmission force in first level clamp $F_{1}$ between the example 3 and 4 . Fig. 10 shows the comparison of the transmission force in second level clamp $F_{2}$ between the example 3 and 4.

Based on the comparison of the transmission force in first level clamp $F_{1}$, the transmission force of the first level clamp $F_{1}$ in example 4 is higher than the it in example 3 in most frequency. When the first level clamp is rigid installed, the transmission force of the first level clamp $F_{1}$ will be higher it in the example which the first level clamp is elastic installed. However, comparing the transmission force of second level clamp $F_{2}$, it is found that the transmission force of the second level clamp $F_{1}$ in example 4 is almost one order of magnitude smaller than it in example 3 . It indicates that installation the first level clamp rigidly not only can improve the effect of impeding the propagation of low frequency vibration wave $(f<50 \mathrm{~Hz})$, but also can lead to the transmission force mainly concentrates on the first level clamp, which greatly reduces the transmission force in the subsequent clamp. Therefore, Using this result, it is possible to concentrate the transmission force on the first level clamp of pipe, and to reduce the transmission force in the subsequent clamp. The limited resource could be used to control the vibration transmission at the first level clamp of pipe to obtain a more optimized plan.

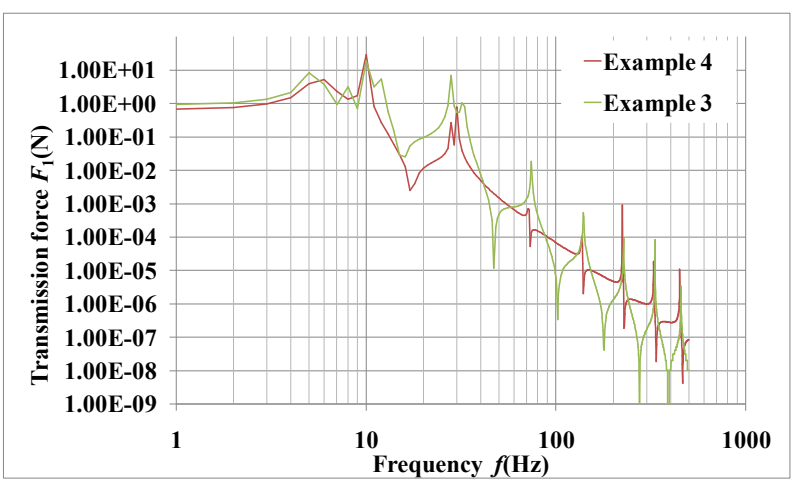

Fig. 9. Comparison of the transmission force of first level clamp $F_{1}$ between example 3 and 4.

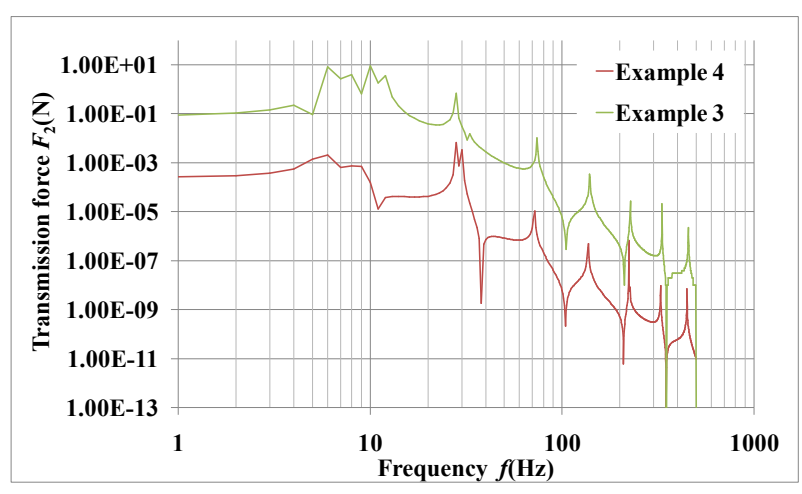

Fig. 10. Comparison of the transmission force of second level clamp $F_{2}$ between example 3 and 4 .

\section{Conclusion}

The performance of flexible connection with vibration isolation mass in impeding vibration wave propagation is studied in this paper. Based on the finite element method, the influences of vibration isolation mass and rotational inertia on the vibration wave propagation in pipe were discussed, and the following conclusions are obtained.

(1) The isolation effect of flexible connection with vibration isolation mass is better than the flexible connection with not vibration isolation mass.

(2) Vibration isolation mass can obviously impede the propagation of vibration wave which frequency higher than $100 \mathrm{~Hz}$. Meanwhile, when the density of pipe remain unchanged and the mass of vibration isolation mass lower than a certain value, the heavier the isolation mass, the better the isolation effect. but when the mass of vibration isolation mass higher than a certain value, the isolation effect is not changed obviously. Therefore, in practical application of vibration isolation mass, it should be reasonable decided the mass of vibration isolation mass. Considering comprehensively, the plan that setting the mass of vibration isolation mass as heavy as possible on the premise of not exceeding the weight restriction of the ship structure may be not a global optimal solution in most case.

(3) The rotational inertia of vibration isolation mass has a significant effect on impeding vibration wave propagation in pipe. Therefore, in practical application of vibration isolation mass, it should be increased the 
rotational inertia of vibration isolation mass as much as possible with a limited mass of vibration isolation mass.

(4) When the first level clamp of pipe installed rigidly, it cloud further improve the isolation effect of vibration isolation mass in low frequency bands. In addition, the exciting force transferred by the clamps after the first leve1 clamp of pipe are obvious reduced.

\section{References}

1. Y Shi, X Zhu, Z.P Hu. Influence of quadrate steel beam with vibration-damping structure on vibration of combined plates. Journal of Naval University of Engineering, 15, 2: 45-49, (2003, in Chinese)

2. Y. Shi, X. Zhu, R.Q Liu. Analysis and experimental research on the role of quadrate steel beam in isolation vibration wave. Shipbuilding of China, $\mathbf{4 5}$, 2:36-42, (2004, in Chinese)

3. Y.Y Liu. The rigid sound insulation characteristics study on obstructs. Ship \& Ocean Engineering, 35, 4: 31-34,(2006, in Chinese)

4. L. Cremer, M. Heckl, E.E Ungar. Structure Borne Sound. Second Edition. Berlin: Springer-Verlag, (1999)

5. X.L Yao, D.J Qian, A.M Zhang, F. Ji. Survey and Tendency of Study on Rigid Vibration-isolating
Technique. Chinese Journal of Ship Research, 3, 5:1-12, (2008, in Chinese)

6. J.H Liu, X.D Jin, Z Li. Impediment to structureborne sound propagation from vibration isolation mass. Journal of Shanghai Jiaotong University, 37, 8, 1201-1204, (2003, in Chinese)

7. Y.S Lin, W.G Wu. Method for solving problems about impeding structure-borne sound transmission from blocking masses and wave impedance characteristics. Journal of Huazhong University of Science and Technology, 43, 10:30-36, (2015, in Chinese)

8. Z.J Yang, Y.C Yang, Y.P Shen, C.L Chen. Experimental Research on Vibration Isolation Mass in Vibration Damping and Noise Reduction of Pipe Conveying Fluid in Ocean Engineering. Ship Engineering, 36, 1: 206-209, (2014, in Chinese)

9. L He, S.P He. Application of transmission loss for flexural wave of infinite rod with blocked mass. Technical Acoustics, 2: 153-157, (2007, in Chinese)

10. C Wang. Research on vibration and noise reduction of ocean engineering pipelines. Ocean university of China, (2015) 